\title{
DEUX ANNÉES D'EXPÉRIMENTATION SUR LA PROTECTION DU BOIS DES RUCHES
}

\author{
Note Technique \\ PAR \\ J. FRESNAYE
}

Station expérimentale d'apiculture, Montfavet.

\section{I. - Introduetion}

L,es méthodes de protection des bois exposés aux agents naturels sont nombreuses et variées. Les données essentielles sur leur composition, procédé d'application, prix de revient, inconvénients, ont été fournies dans une précédente note $(\mathrm{I})$. On trouvera ici les résultats des premières expériences de plein air sur quelques revêtements protecteurs. Bien qu'elles n'aient porté que sur deux années, elles permettent déjà un certain nombre de conclusions.

\section{II. - Conditions des expériences sur les ruches}

L'expérience porte sur deux ruches pour chaque revêtement ou méthode de protection ce qui nous permet de grouper les différents revêtements dans un même rucher. Ces ruches sont donc soumises aux mêmes conditions météorologiques.

Nous avons choisi l'emplacement du rucher pour son climat assez rude; très chaud et ensoleillé l'été, humide et froid dans les périodes hivernales. Ces conditions se trouvent réunies, dans notre région, sur les pentes nord du Massif du Lubéton, à cinq cents mètres d'altitude, aux environs d'Apt. Une haie épaisse protège les ruches des vents dominants.

Ces ruches ne suivent pas la transhumance habituelle de l'apiculture provençale. Les expériences en rucher fixe constituent un préliminaire obligatoire pour la compréhension des dégradations dues à la transhumance qui seront donc l'objet d'autres études. L'état des revêtements est apprécié visuellement; en effet nous ne disposons pas d'appareil permettant de mesurer la dureté ou la résistance d'un enduit.

Nous tenons compte de l'aspect général : craquelures, écaillage, cloquage, fendillement, farinage, pulvérulence et autres états de dégradation.

Annales des Abeilles. - ig6o. 


\section{III. - Résultats des deux premières années de l'expérience}

On peut penser que les notations de la prenière année manquent d'intérêt. Cependant des indices significatifs permettent déjà les plus grandes réserves au sujet des qualités préservatives de certains revêtements.

Les résultats obtenus la det1xième année ne sont pas définitifs mais d'ores et déjà il est possible d'éliminer certains revêtements qui, au bout de ce laps de temps se trouvent dans un état de dégradation avancé. Ainsi resteront seuls en. compétition les revêtements qui se sont bien comportés pendant les deux premières années. Les notations de ces deux années permettent de suivre l'évolution des différents types de dégradation.

\section{A. - Ruches témoins, sans revêtement (Ruches $n^{\circ} 25^{-26}$ ).}

Première année. Le bois est en mauvais état tout au moins superficiellement. La saison étant humide, il y a des moisissures aux assemblages, surtout au Nord. Le bois se fendille et joue (on emploie aussi le terme "travaille" qui désigne également le gonflement ou le retrait, dus aux variations de l'humidité atmosphérique et aux changements de temps). La teinte devient plus foncée et inesthétique.

Deuxième année. Le bois " travaille " énormément. Les parois constituées d'une seule planche sont fendues, celles qui sont constituées de plusieurs morceaux ont leurs " languettes" presque sorties des " rainures") (on désigne par ces noms les parties mâles et femelles permettant l'assemblage de deux planches juxtaposées). Les assemblages des coins des ruches sont également disjoints. Les plateaux de sol ont particulièrement souffert et se déforment. Les parois présentent de grandes taches noires dues aux attaques cryptogamiques.

\section{B. - Peintures employées seules.}

I. - Peinture a L'Hutle, PRemière gualití (ruches no i5-I6).

Première année. - La couche de peinture est en très bon état et le bois n'a pas joué. On note seulement des craquelures fines et superficielles; elles ne traversent pas toute l'épaisseur de la couche de peinture. Ce type de craquelures est généralement dû au fait que la première couche ou couche d'impression, contient autant d'huile ou plus que les couches de finition alors que son pourcentage d'huile devrait être plus faible.

Deuxième année. - Bon état du revêtement dans l'ensemble. Les craquelures superficielles précédemment signalées n'ont subi aucune aggravation. Un léger farinage se produit à la surface de la peinture : des particules du revêtement restent sur les mains. Le "farinage "ot désagrégation progressive du film n'est pas un défaut grave lorsqu'il reste peu important comme dans le cas présent (nous verrons pourquoi plus loin). Le ternissement du film est également d $\hat{\imath}$ au farinage.

\section{2. - Peinture a L'huile,}

PIGMENT ALUMiniUm (APPligUÉE A LA bROSSE) (ruches no-3-4).

Première année. - La couche de peinture est en très bon état, elle ne présente aucune détérioration : le film couvre mieux le bois, il est plus épais et plus lisse que lorsque la même peinture est appliquée au pistolet. Le bois ne travaille presque pas. (Aucun revêtement ne peut d'ailleurs arrêter complètement le gonflement ou le retrait de ce matériau très hygroscopique.) 
Deuxième année. - 'Très bon état, aucun défaut ou dégradation. II n'apparaît aucune trace d'attaque cryptogamique ou d'insectes prédateurs. Pas de farinage, la peinture est dure, lisse et brillante.

Le pigment métallique aluminium assure une bonne réverbération des radiations solaires. L'aspect des ruches est agréable à l'œil. Nous retiendrons ce rezêtement comme l'un des meilleurs en apiculture actuellement.

\section{3. - Pennture a i, huile pigment aluminium (APPLIQUÉE AU PISTOLE'T) (ruches no $3 \mathrm{I}-32$ ).}

Première année. - La couche de peinture est en très bon état. Cependant, les parties du bois "en bout", ne sont pas suffisamment couvertes par la peinture ce qui nuira certainement à la longévité du revêtement. Le film protecteur est trop mince.

Deuxième année. - Bon état du revêtement dans l'ensemble, la couche de peinture, trop mince, laisse apparaitre un début de noircissement principalement aux assemblages et sur le bois « en bout ». L'application au pistolet n'est pas à condamner pour autant, mais il faudrait prévoir une ou deux couches supplémentaires de peinture pour obtenir un bon résultat. Il est préférable d'appliquer la couche d'impression à la brosse.

$$
\text { 4. - Peinture vinyligue: (ruche no } \mathrm{I}-2 \text { ). }
$$

Première année. - Le revêtement est en très bon état et ne présente aucune détérioration. Le bois, bien protégé, n'a pas joué et ne s'est pas fendillé.

Le seul inconvénient à ce stade, inconvénient mineur, provient de l'aspect mat et légèrement rugueux de la peinture qui de ce fait retient davantage les salissures faites par les mains, la terre, les taches grasses, qu'un film très lisse et brillant.

Deuxième année. - Le revêtement est en bon état et il n'y a pas d'augmentation notable des salissures précédemment signalées. Ces salissures seront probablement très accentuées lorsque les ruches ainsi recouvertes devront transhumer. Un léger fendillement apparaît par endroits à la surface du bois. Il est à craindre qu'il ne facilite l'attaque des divers agents de dégradation à brève échéance.

\section{5. - PEINTURE GI.YCíROPHTAIIQUE PREMière QUALiTí (ruches $1^{\circ}$ 23-24).}

Première année. - L'état du revêtement est médiocre. Il se soulève par petites plaques et s'écaille, principalement près des joints et assemblages du bois. Cette dégradation précoce nous a beaucoup surpris, les peintures glycérophtaliques étant, de l'avis général, les revêtements les plus sûrs et les plus durables à l'extérieur. Elle s'est d'ailleurs produite dès les premiers mois de l'expérience et nous avons pu la signaler dans notre précédente note (I). Il est souvent utile de diluer les peintures glycérophtaliques avant leur emploi. Des couches trop épaisses appliquées en une fois sont nuisibles à une bonne tenue de ces peintures et il est préférable d'appliquer une couche supplémentaire pour obtenir un revêtement de l'épaisseur désirée.

Deuxième année. - Le revêtement est en mauvais état et l'écaillage est assez important. Nous restons sceptiques quant à la valeur de cette peinture pour la protection des ruches et surtout des ruches de pin, même en tenant compte de l'élimination de certains défauts d'application possibles. Ces défauts, 
mineurs dans le cas présent car il s'agissait de peintures prêtes à l'emploi, en boîtes de peu de volume et n'ayant pas vieilli, ne nous semblent pas expliquer un tel état de dégradation.

\section{6. - Peinture celiulosigue (ruches no 13-I4).}

Première année. - La peinture est en très mauvais état. L'écaillage est important, en fines paillettes, sur toute la surface de la ruche. Toute la peinture tombe en poudre fine au moindre frottement.

Deuxième année. - Le revêtement est entièrement détruit. Il est inutile de continuer l'expérience plus longtemps.

7. - Peinture glycérophtalique deuxième qualité (ruches no 6i-62).

Première année. -- Cette peinture, moins onéreuse, donc de qualité légèrement inférieure à celle des ruches $\mathrm{n}^{\circ} 23-24$, avait peu de chance de mieux se comporter que celle-ci. En effet, on constate un écaillage très important sur toute la surface de la ruche ce qui corrobore le résultat obtenu sur les ruches $\mathbf{n}^{0}$ 23-24 et contribue à condamner ce type de peinture sur ruches en bois de pin.

\section{c. - Fongicides.}

\section{I. - Carbonyle (ruches no 5-6).}

Première année. - Le bois est en très bon état, propre et sans moisissure. On note un début d'éclaircissement de la teinte du revêtement. L'aspect est moins esthétique que celui des ruches peintes.

Deuxième année. - Bon état, aucune attaque de champignons ou d'insectes. La teinte s'éclaircit notablement. Un fendillement en beaucoup d'endroits et quelques fentes très profondes nous montrent que le carbonyle ne protège que très incomplètement le bois.

\section{2. - Fongicide chloronaphtaléNiQue (ruches $n^{\circ} 7-8$ ).}

Première année. - - Bon état du bois dans l'ensemble. Il faut cependant tenir compte d'un fendillement partiel (d'ailleurs à peine perceptible) sur différentes planches. Les clous sont attaqués par la rouille. Ce produit est un fongicide, non un revêtement.

Deuxième année. - Aucune attaque fongique ou entomologique, mais le bois se fend en beaucoup d'endroits, les assemblages se disjoignent. Le bois " travaille " beaucoup et les ruches ressemblent à ce point de vue aux ruches sans revêtement. A ne pas employer sans revêtement complémentaire.

\section{D. - Peinture avec sous-couche fongicide ou hydrofuge.}

I. - Peinture a l'huile pigment aluminium,

SOUS-COUCHE FONGICIDE CHLORONAPHTALÉNIQUE (ruches no II-I2).

Première année. - Le revêtement est en très bon état, le bois est également exempt de dégradation.

Deuxième année. - La couche de peinture est en assez bon état dans l'ensemble. Cependant, le film n'adhère pas au bois par endroits et s'effrite facilement au moindre frottement. Le fongicide employé en sous-couche semble donc à longue échéance, être incompatible avec la peinture et accélère sa dégradation. Ce traitement est donc à proscrire et les frais qu'il occasionne sont superflus. 


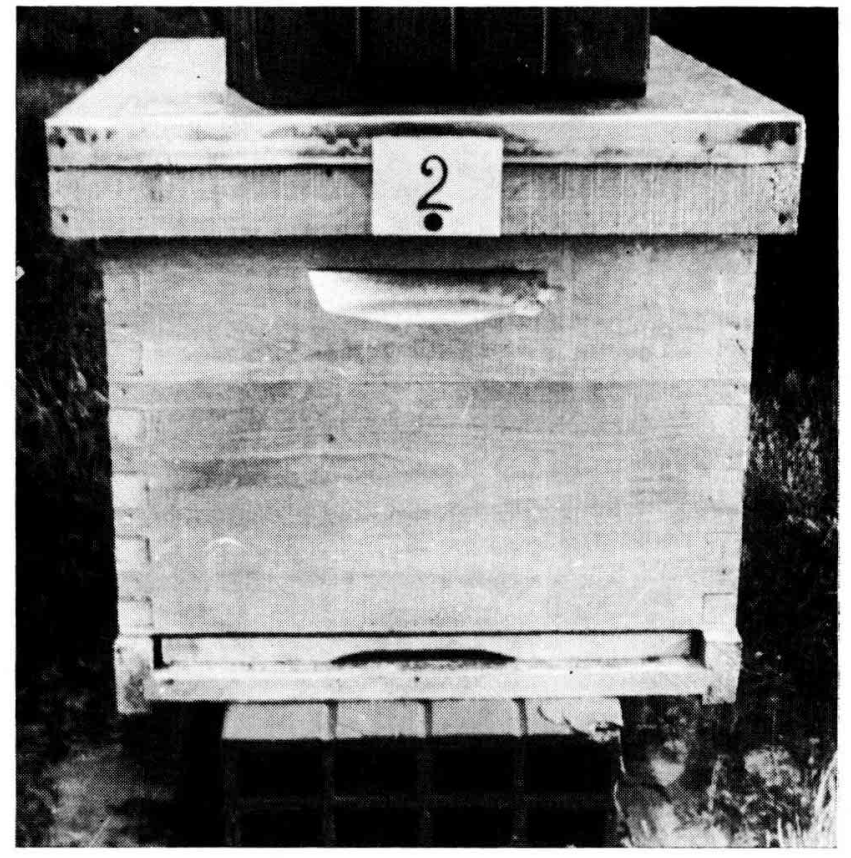

Rercelement $\imath^{0}$ 2. - Peinture aluminium au pinceau (ruches 3 et 4 ).

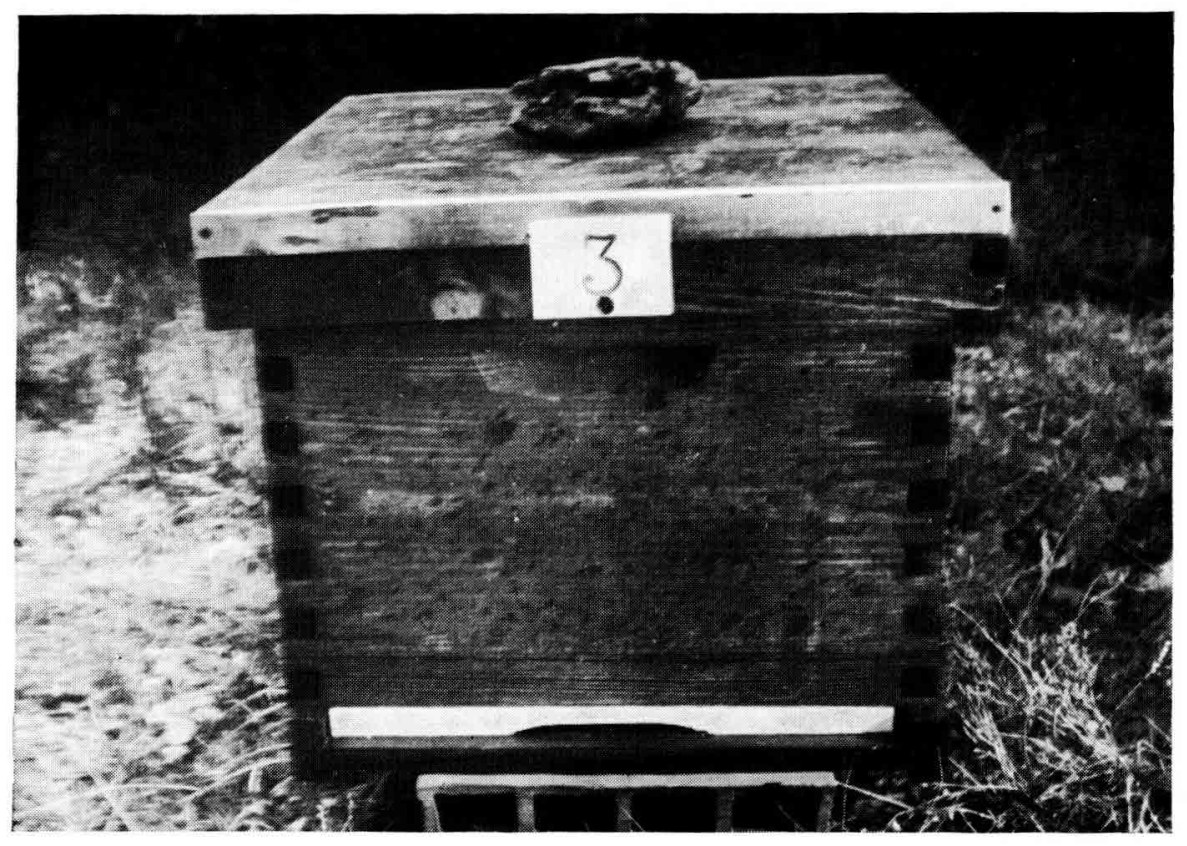

Revátenent $n^{0}$ 3. - Carbongle + huile de lin (ruthes 5 et (o). 


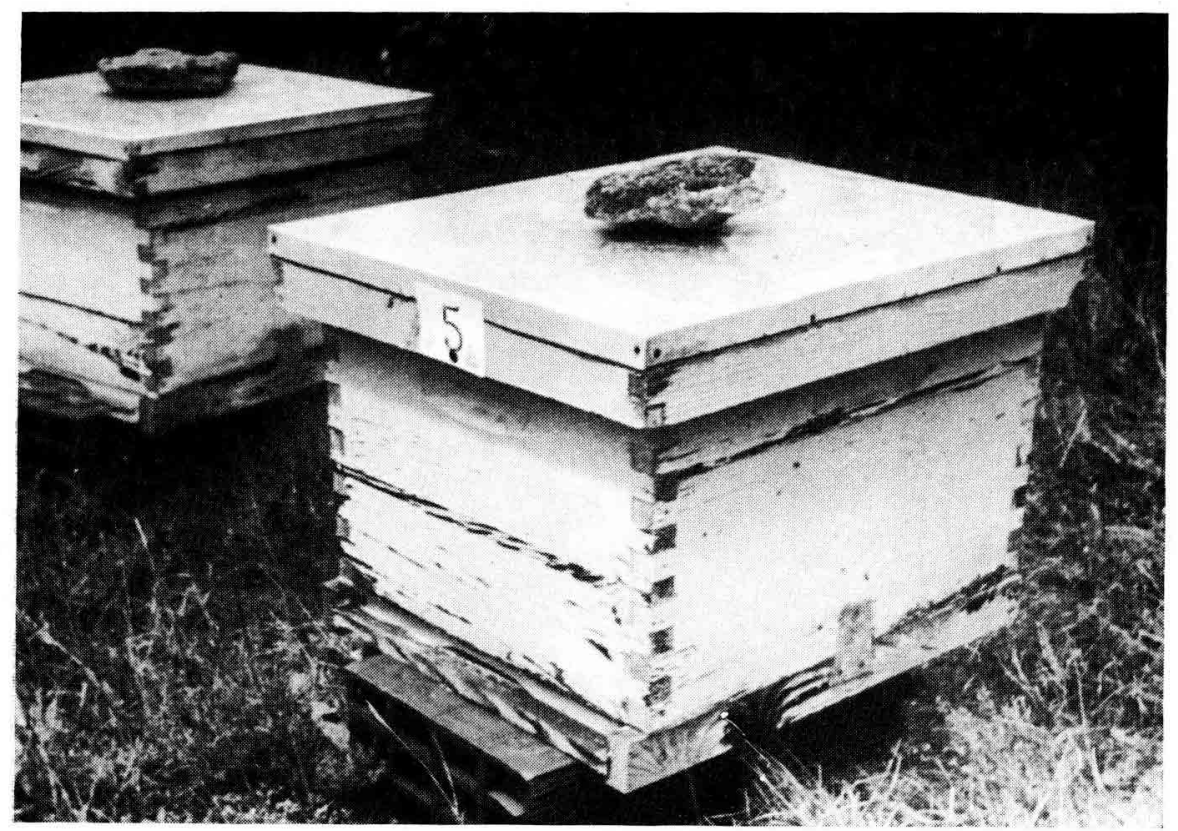

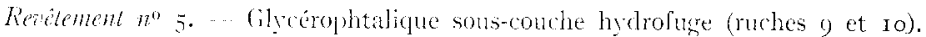

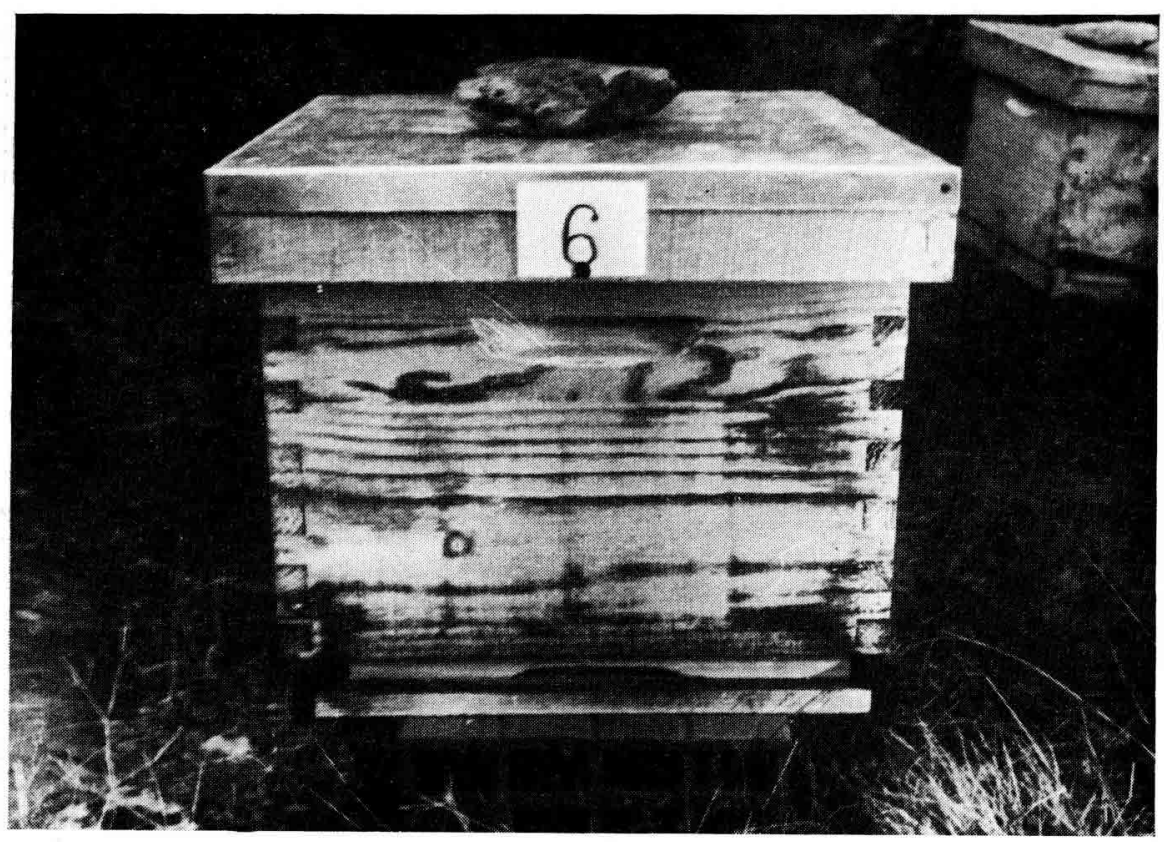

Revitement to 6 . - P'einture aluminium sous-conche fongicide (ruches i s et 12 ). 


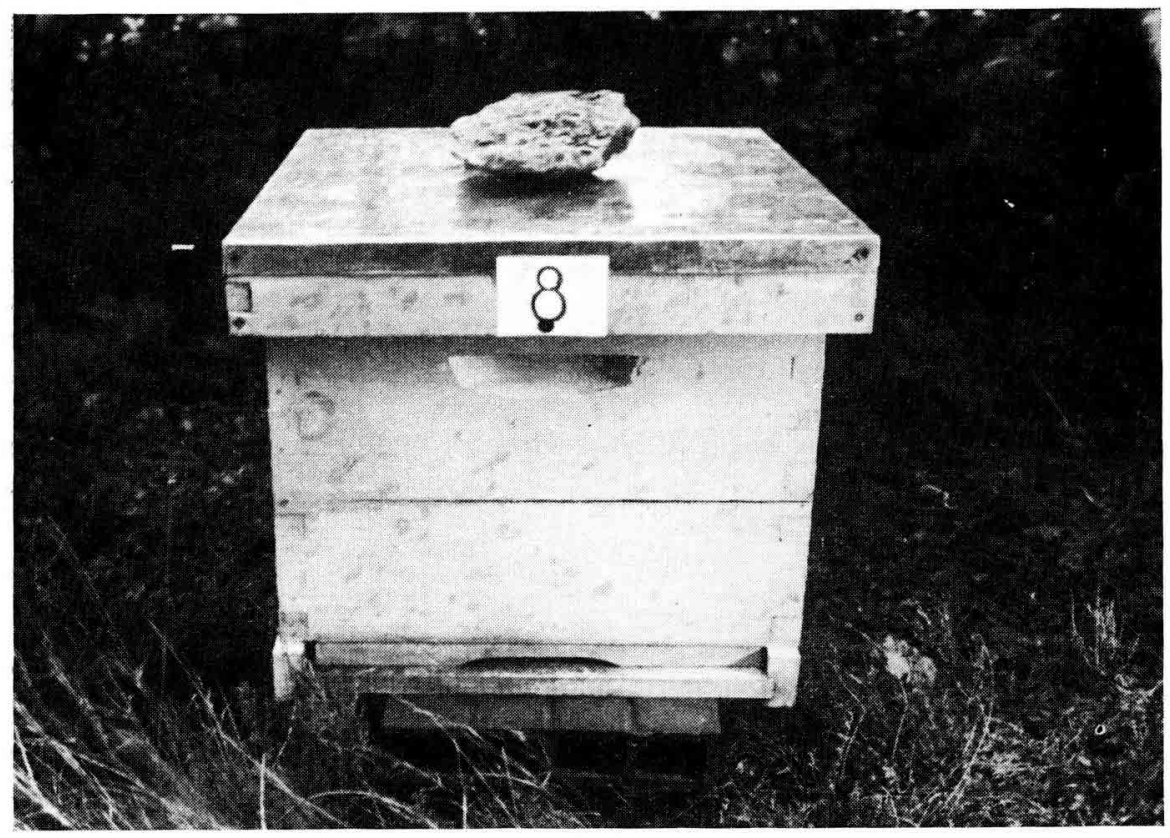

Rectement $n^{0}$ 8. - P'tinture à l'huile (ruches 15 ct 16).

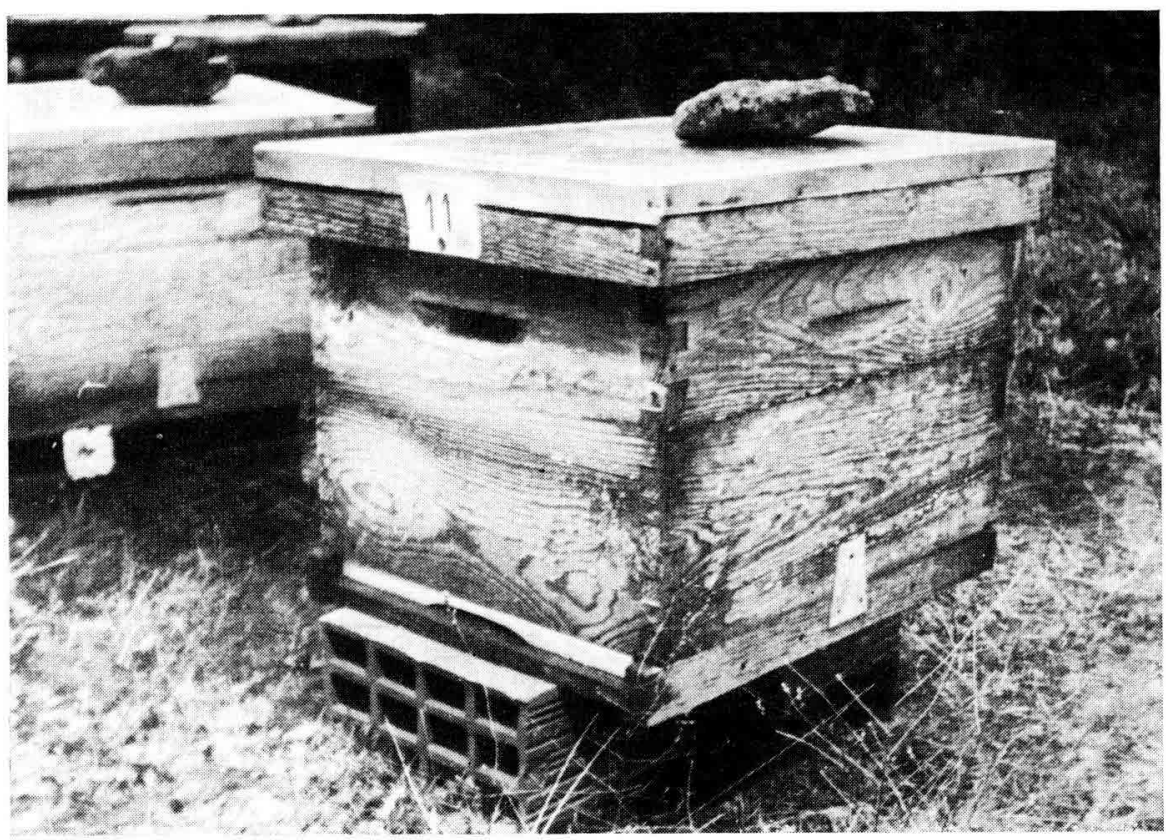

Revilnent $\|^{6}$ I T. - Jarafine (ruches $2 \mathrm{I}$ at 22). 


\section{2. - Peinture glycérophtaligue SOUS-COUChe hydrofuge (ruches $\mathrm{n}^{\mathbf{0}}$ 9-IO).}

Première année. - - L'état du revêtement est médiocre. Il se décolle du bois et s'écaille. Bien que l'hydrofuge soit prévu pour ce genre de peinture il se peut qu'il soit responsable de cet écaillage.

Deuxième année. - Le revêtement est en très mauvais état. L'écaillage est important et se fait par grandes plaques de plusieurs centimètres carrés.

On ne peut incriminer la sous-couche hydrofuge seule : on a vu l'état des autres essais avec des peintures glycérophtaliques. Malgré tout la dégradation est ici plus rapide encore.

Nous inciterons donc les apiculteurs à beaucoup de circonspection lorsqu'ils voudront réaliser des associations fongicide-peinture ou hydrofuge-peinture, et à s'assurer au préalable, de la compatibilité des deux produits.

\section{E. - Vernis.}

\section{I. - Vernis polyuréthane a Catalyser (ruches no 29-3o).}

Première année. - Les ruches ont un bon aspect dans l'ensemble, le vernissage est agréable à l'œil. Un début d'écaillage se produit le long de petites fentes du bois. Cette dégradation est inquiétante pour un revêtement qui n'a qu'une année d'expérimentation.

\section{2. - VERNIS GLYCÉROPHTALIQUE (ruches no 59 -60).}

Première année. - Le revêtement est dans un état médiocre. Un léger écaillage met le bois à nu en divers endroits. Sous le vernis, on aperçoit par transparence de grandes taches noirâtres indice d'une importante attaque fongique. Le mycélium qui cause ces taches développe ses fructifications et supprime l'adhérence du revêtement, par ailleurs la dégradation est excessive pour une exposition d'un an seulement aux intempéries.

\section{F. - Paraffine seule ou avec sous-couche.}

\section{I. - Paraffine (ruches $\mathrm{n}^{0}$ 2I-22).}

Première année. - La couche de paraffine est en très mauvais état. Elle se décolle par plaques importantes, laissant le bois à nu. Cependant, dans les parties ainsi découvertes, le bois n'a subi aucune attaque. Il est protégé par la paraffine qui a pénétré dans ses pores. La ruche a mauvais aspect dans 1'ensemble.

Deuxième année. - L'écaillage de la couche de paraffine est presque total. Malgré l'écaillage il reste de la paraffine sur le bois, on la décèle en frottant un objet dur sur les parties écaillées.

\section{2. - Paraffine avec sous-couche carbonyle (ruches no i7-I8).}

Première année. - Sur ces ruches le paraffinage est pratiqué après deux mois de séchage du carbonyle appliqué à la brosse. La paraffine s'écaille 
beaucoup. Le bois n'a subi aucune dégradation étant protégé par le carbonyle et la paraffine qui a pénétré dans les pores. Les ruches ont très mauvais aspect.

Deuxième année. - Écaillage presque total de la couche de paraffine. Bon état du bois. Contrairement aux ruches dont le carbonyle est le seul revêtement il n'y a aucun éclaircissement de la teinte, les ruches restent très noires ce qui leur donne, joint à l'écaillage de la couche de paraffine, un aspect très déplaisant.

\section{3. - Paraffine avec sols-covche fongicide chloronaphtalénioue} (ruches $\mathrm{n}^{\mathrm{O}} \mathrm{I} \mathrm{9}^{-20}$ ).

Première année. - 'Très mauvais état de la couche de paraffine. Décollement sur toute la surface du bois. 'Très mauvais aspect général, mais aucune dégradation du bois.

Deuxième année. - Le bois est en bon état. Êcaillement total de la couche de paraffine.

Dans cette série d'expériences, le paraffinage a été pratiqué comme les apiculteurs le font généralement : bref trempage du bois dans un bain de température trop basse et variable. Ce procédé est à proscrire. Nous en avons déjà parlé ailleurs et nous reviendrons sur ce point important dans un chapitre annexe.

\section{G. - Huile de lin avec sous-couche carbonyle (ruches $n^{0} \quad 27-28$ ).}

Première année. - Le bois est en très bon état. La teinte, à l'origine très foncée du carbonyle, s'éclaircit per1 à pe1. On ne décèle aucune trace d'application d'huile de lin, et aucune différence avec les ruches qui ont seulement reçu du carbonyle comme revêtement.

Deuxième annéc. - I es ruches sont en très bon état. I a teinte s'éclaircit encore. La protection supplémentaire offerte par l'huile de lin réduit le fendillement du bois bien (qu'il ne reste aucune trace apparente de son application. Pour assurer une protection plus efficace encore il serait nécessaire d'appliquer une couche d'huile de lin chande tous les deux ans.

\section{H. - Vernis plastique.}

\section{I. - Vernis piastigue EMPloyí SLIL (ruches $11^{0} 428-429$ ).}

Première année. - Le revêtement est en très manvais état, bien que l'essai ne soit commencé (que depuis 6 mois. Il se sépare du substrat et tombe en pellicules souples et de grande taille. Le décollement se produit principalement près des fentes du bois. Ce produit est à utiliser uniquement sur une peinture faisant fonction de sous-couche.

\section{2. - VERNIS PLASTIQUE SUR PEINTURE A L'HUILE ET PIGMENT ALUMINIUM EX SOUS-COUCHE (ruches $n^{\circ} 426-427$ ).}

Première année. - Le terme sous-couche désigne généralement la couche appliquée directement sur le subjectile, c'est la couche d'impression sur les 
parties absorbantes (bois), la conche primaire sur les parties non absorbantes (fer) (2). Elle peut être en outre hydrofuge, fongicide ou insecticide. Dans le cas présent il s'agit d'un revêtement déjà complet, comportant couches d'impression et de finition. Le vernis plastique est un complément qui doit assurer la protection de ce reiêtement.

L'essai n'a débuté que depuis six mois, les résultats sont donc peu probants actuellement. Les ruches sont en très bon état, l'application du plastifiant est peu décelable lors d'un examen superficiel.

\section{IV. - Évolution de la dégradation des divers revêtements}

Nous avons donné dans le chapitre précédent les notations détaillées de l'état des divers revêtements expérimentés. Si ces descriptions permettent de se faire une idée des différents défauts ou qualités d'un revêtement, un tableau synoptique permettra de les comparer entre eux. Pour être plus clairs nous avons réduit à quatre échelons les notations désignant la valeur des revêtements.

\begin{tabular}{|c|c|c|c|c|c|c|}
\hline Revitement & $\begin{array}{l}\text { No des } \\
\text { ruches }\end{array}$ & Année & $\begin{array}{l}\text { Très bon } \\
\text { etat }\end{array}$ & Jon état & $\begin{array}{l}\text { Etat } \\
\text { mécliocre }\end{array}$ & $\begin{array}{l}\text { 'Très } \\
\text { mauv. état }\end{array}$ \\
\hline \multirow{2}{*}{ Sans revêtemt'nt } & \multirow{2}{*}{$25-26$} & $\mathrm{I}^{\mathrm{re}}$ année & & & $\mathrm{X}$ & \\
\hline & & ze amnée & & & & $\mathrm{X}$ \\
\hline \multirow{2}{*}{$\begin{array}{l}\text { Peinture à l'huile } \\
\text { re qualité }\end{array}$} & \multirow{2}{*}{ I6-I 5} & $I^{\text {re }}$ amnée & $\mathrm{X}$ & & & \\
\hline & & $2^{e}$ annce & & $\mathrm{X}$ & & \\
\hline Peinture à l'huile & \multirow{2}{*}{$3-4$} & I $^{\text {re }}$ année & $\mathrm{X}$ & & & \\
\hline (appliquée à la brosse) & & ze année & $\mathrm{X}$ & & & \\
\hline Peinture a l'huile & \multirow{2}{*}{$31 \cdot 22$} & $1^{\text {re }}$ anmée & $\mathrm{X}$ & & & \\
\hline (ap)lique au pistolet) & & $2^{e}$ annes & & $\mathrm{X}$ & & \\
\hline \multirow{2}{*}{ l'einture vinylque } & \multirow{2}{*}{$I-2$} & I $^{\text {re }}$ atumés & $\mathrm{X}$ & & & \\
\hline & & $z^{e}$ :amué & & $\mathrm{X}$ & & \\
\hline \multirow{2}{*}{$\begin{array}{l}\text { Peinture grlycérophtalique } \\
\text { re qualité }\end{array}$} & \multirow{2}{*}{$2.3-24$} & I $^{\text {re }}$ asmée & & & $\mathrm{X}$ & \\
\hline & & 2 amnée & & & & $x$ \\
\hline \multirow{2}{*}{ Peinture cellullosique } & \multirow{2}{*}{$\mathrm{I} 3-\mathrm{I} 4$} & I $^{\text {re }}$ ammee & & & & $\mathrm{X}$ \\
\hline & & $z^{\text {e }}$ ammée & & & & $\mathrm{X}$ \\
\hline \multirow{2}{*}{$\begin{array}{c}\text { Peinture glycérophtalique } \\
2^{6} \text { qualite }\end{array}$} & \multirow{2}{*}{$6 \mathrm{I}-62$} & Ire $^{\text {ammee }}$ & & & - & $\mathrm{X}$ \\
\hline & & $2^{\mathrm{e}}$ annés & 110 & Éoulée & & \\
\hline \multirow{2}{*}{ Carbonyle } & \multirow{2}{*}{$5 \cdot 6$} & I $^{\mathrm{re}}$ anmée & $\mathrm{X}$ & & & \\
\hline & & $2^{e}$ année & & $X$ & & \\
\hline \multirow{2}{*}{$\begin{array}{c}\text { Fongicide } \\
\text { Chloronaphtalénique }\end{array}$} & \multirow{2}{*}{$7-8$} & I $^{\text {re }}$ anmcie & & $\mathrm{X}$ & & \\
\hline & & $2^{e}$ annnée & & & $\mathrm{X}$ & \\
\hline
\end{tabular}




\begin{tabular}{|c|c|c|c|c|c|c|}
\hline Revêtements & $\begin{array}{l}\text { No des } \\
\text { ruches }\end{array}$ & Année & $\begin{array}{l}\text { Très bon } \\
\text { état }\end{array}$ & Bon état & $\begin{array}{l}\text { État } \\
\text { médiocre }\end{array}$ & $\begin{array}{l}\text { Très } \\
\text { mauv. état }\end{array}$ \\
\hline $\begin{array}{l}\text { Peinture à l'huile pigment } \\
\text { Aluminium sous-couche }\end{array}$ & $1 \mathrm{I}-\mathrm{I} 2$ & $1^{20}$ année & $x$ & & & \\
\hline longicide choronaphtale. & $11-12$ & $z^{e}$ année & & & $\mathrm{X}$ & \\
\hline \multirow{2}{*}{$\begin{array}{l}\text { Peintureglycérophtalique } \\
\text { sous-couche hydrofuge }\end{array}$} & \multirow{2}{*}{$y-10$} & J $^{\mathrm{re}}$ année & & & $x$ & \\
\hline & & $2^{e}$ année & & & & $\mathrm{X}$ \\
\hline \multirow{2}{*}{$\begin{array}{l}\text { Vemis poiyurethane } \\
\text { a catalyser }\end{array}$} & \multirow{2}{*}{$20 \cdot 30$} & ${ }^{\text {re }}$ innée & & & $\mathrm{X}$ & \\
\hline & & $2^{n}$ année & non & écoules & & \\
\hline \multirow{2}{*}{ Vernis glycérophtalique } & \multirow{2}{*}{$=0-60$} & Ire amnée & . ..-- & & $x$ & \\
\hline & & It anméc & non & écoultéx & & \\
\hline \multirow{2}{*}{ Paraftine } & \multirow{2}{*}{$21-22$} & $\mathrm{I}^{\mathrm{re}}$ année & & & & $\mathrm{x}$ \\
\hline & & $2^{\mathrm{e}}$ annce & & & & $\mathrm{X}$ \\
\hline Paraffine & \multirow{2}{*}{$I 7-1 S$} & $I^{r \prime}:$ :1nnée & & & & $\mathrm{X}$ \\
\hline sous-couche (arbonyle & & $2^{*}$ : annes & & & & $\mathrm{X}$ \\
\hline \multirow{2}{*}{$\begin{array}{c}\text { Paraffine sous-couche } \\
\text { Fongicide } \\
\text { chloronaphtalénique }\end{array}$} & \multirow{2}{*}{$19-20$} & Ire innè & & & & $\mathrm{X}$ \\
\hline & & $z^{e}$ annéz & & & & $\mathrm{X}$ \\
\hline \multirow{2}{*}{$\begin{array}{l}\text { IIuile de lin } \\
\text { sou-couche Carbonyle }\end{array}$} & \multirow{2}{*}{$27-28$} & $\mathrm{I}^{\mathrm{re}}$ annés & $x$ & & & \\
\hline & & $z e$ anné & $x$ & & & \\
\hline \multirow{2}{*}{ Plastifiant } & \multirow{2}{*}{$42+4+29$} & Ire année & & & & $\mathrm{X}$ \\
\hline & & ze annéé & non & écoulti & & \\
\hline \multirow{2}{*}{$\begin{array}{l}\text { Plastifiant sous-couche } \\
\text { peinture a l"hule } \\
\text { pimment ahminium }\end{array}$} & \multirow{2}{*}{$+26-+27$} & $\mathrm{I}^{\mathrm{re}}$ amnée & $\mathrm{X}$ & & & \\
\hline & & ze année & 1101 & 'écortée & & \\
\hline
\end{tabular}

\section{Conclusion}

Les conclusions à tirer de ces expériences sont encore provisoires car les essais doivent se poursuivre pendant plusieurs années encore pour être probants.

I $^{0}$ Les ruches sans revêtement, et n'ayant jamais reçu aucun revêtement, se déforment : le bois se fend rapidement et est l'objet d'attaques fongiques et entomologiques qui rendent les ruches inutilisables dans un bref délai.

$2^{\circ}$ Les peintures à l'huile de prenière qualité, résistent correctement aux divers agents de dégradation pendant les detux premières années de leur emploi; nous sarons par aillewrs que leur bonne tenue dipasse rarement trois ans.

$3^{\circ}$ Les peintures à l'huile arec pigment aluminium simblent être actuellement lee revêtements les plus cificaces. Si ce revêtement est appliqué au pistolet il est nécessaire de poser quatre ou cinq couches successives pour obtenir une protection suffisante. L'application à la brosse est préférable car elle permet d'imprégner au maximum le bois en bout et les divers assemblages. Les deux premières années d'essai ne montrent aucune dégradation de la peinture. 
$4^{\circ}$ Les peintures vinyliques ont une bonne tenue à l'extérieur. Elles sesalissent facilement et la transhumance nuira à leur esthétique.

$5^{\circ}$ Les peintures glycérophtaliques sont toutes en mauvais état. On devra donc en éviter l'emploi sur les ruches de pin.

$6^{\circ}$ Le carbonyle est un puissant fongicide mais il ne protège pas le bois. contre les variations dimensionnelles. L'application d'huile de lin chaude pos-. térieurement au traitement au carbonyle réduit cet inconvénient et les résultats obtenus sont alors très intéressants surtout si l'on considère la facilité d'application et le prix de revient.

$7^{\circ}$ Les fongicides et hydrofuges ne doivent pas s'employer seuls, mais en sous-couche. D'autre part, ils nuisent parfois à la bonne tenue du revêtement de finition. Leur emploi semble done en fin de compte peu rentable car leur compatibilité avec le revêtement terminal est problématique.

$8^{\circ}$ Les vernis que nous avons essayés ne présentent aucun intérêt pour l'apiculture tant par leur mauvaise tenue que par leur prix de revient élevé.

$9^{\circ}$ Les méthodes empiriques de paraffinage employées jusqu'ici sont à proscrire, nous l'avons vu dans nos essais. Il faut pour réussir ce genre de revêtement : $80^{\circ}$.

a) Employer des cires microcristallines, à point de fusion avoisinant

b) Que le bain de cire soit extrêmement chaud, sans toutefois aller jusqu'à la décomposition de la cire.

c) Tremper assez longuement le bois dans le bain pour qu'il s'échauffe et: permette une pénétration plus profonde de la cire.

d) Égoutter à une température élevée, de $30^{\circ}$ à $50^{\circ}$ si possible, afin d'éviter le "glaçage » et d'éliminer la majeure partie de la cire restée en surface, car elle adhère mal au bois et augmente le prix de revient en pure perte.

La protection ainsi obtenue est excellente, et de longue durée, contre. les intempéries et les champignons. Il sera peut-être possible de revenir aux. paraffines raffinées à haut point de fusion lorsque nous aurons obtenu, par. ressuage, l'élimination totale de la couche qui reste en surface.

IO ${ }^{\circ}$ Les vernis plastiques à appliquer sur les peintures assurent peutêtre une protection efficace de celles-ci, nous le saurons ultérieurement. Ils sont d'un prix de revient extrêmement élevé, mais justifié si la protection obtenue atteint ou dépasse tne dizaine d'années.

\section{Appendice}

\section{Causes de la dégradation des revêtements.}

La dégradation des divers revêtements utilisés à 1'extérieur a des causes généralement connues et auxquelles il est possible de remédier plus ou moins. Ces défauts sont de types divers.

\section{Craquelures, ÉcAillage.}

Les craquelures superficielles des peintures à l'huile se produisent lorsque la couche de finition est appliquée sur une couche d'impression insuffisamment sèche. Elles se produisent également lorsqu'une couche de finition " plus courte 
en huile " qu'une couche d'impression. Une peinture insuffisamment agitée avant 1'emploi contiendra trop d'huile en surface et un pourcentage de pigment trop important au fond. Pour les peintures à l'huile, il faut appliquer une couche d'impression à faible pourcentage d'huile, puis les couches de finition contenant de fortes proportions d'huile, 30 à $35 \mathrm{p}$. roo (2).

Les craquelures atteignant le bois se produisent lorsque le revêtement ne peut suivre le " jeu " du bois. Les bords de ces craquelures se soulèvent et c'est le début de l'écaillage. Un excès de siccatif provoque également l'apparition prématurée des craquelures.

Lorsque le film protecteur est trop mince il reste perméable, il n'empêche pas 1'humidité de pénétrer dans le bois et les variations dimensionnelles qui s'ensuivent causent la dégradation de la peinture et du bois.

L'écaillage est causé par un manque d'adhérence du revêtement au substrat ou des différentes couches d'un revêtement entre elles. Ce manque d'adhérence peut avoir différentes raisons.

Le bois a été souillé par des taches de matières grasses, de terre, de poussières. Les bois à peindre seront manipulés avec des mains propres et on leur évitera les contacts gras.

Le bois est trop humide. Les peintures adhèrent toujours moins sur un bois qui contient un pourcentage d'humidité trop élevé lors de l'application du revêtement, ou qui se trouve ensuite constamment placé dans une atmosphère humide.

Le bois a été traité. Les traitements par des produits fongicides, insecticides, ignifuges, hydrofuges, nuisent parfois à l'adhérence des revêtements de finition. Nous en avons signalé des cas dans nos expériences. Il faudrait faire des essais pour s'assurer de la compatibilité de ces traitements avec les peintures employées, mais ces essais pour être probants devraient durer six mois ou un an, car la dégradation du revêtement n'est parfois apparente qu'au bout de ce laps de temps.

Les peintures n'adhèrent pas sur un bois contenant des traces de cire ou de paraffine. Un décapage énergique est alors nécessaire.

Le bois a déjà été peint. Il faut éviter de peindre sur un revêtement dont la surface est trop dure ou trop lisse, l'adhérence sera insuffisante. On doit décaper par brûlage ou à l'aide de puissants décapants. Si le film ancien est en bon état on peut se contenter de la poncer pour favoriser l'accrochage.

\section{FARINAGE.}

C'est la désagrégation progressive du film, sous forme d'une poudre qui est entraînée par les frottements, la pluie ou le vent. Le " farinage " entraîne le ternissement du revêtement. Il ne devient un gros défaut que s'il est très important; le bois est alors rapidement mis à nt. S'il est peu important par contre, la réfection périodique du revêtement est facilitée par la réduction de l'épaisseur du film et un simple ponçage suffit pour assurer l'adhérence de la nouvelle couche. Les pigments mal adaptés au type de peinture, ou un trop fort pourcentage de pigment, provoquent le farinage.

\section{Cloques.}

Les cloques n'apparaissent que sur des films souples peu perméables et adhérant mal au bois. Elles se produisent principalement lorsque des bois humides ou résineux sont soumis à une température trop élevée (3). 
Les peintures glycérophtaliques et cellulosiques ont une faible adhérence sur le bois, notamment sur les résineux. Il est nécessaire de les isoler du bois par un "fond dur "pour améliorer leur tenue, ce qui complique le travail et augmente le prix de revient.

\section{RÉFÉRENCES BIBLIOGRAPHIQUES}

(I) Frestaye (J.). - Protection du bois des ruches. Ann. de l'A beille, II (3), $\quad 235-255, \quad$ I959.

(2) BERnARd (E.). - Peinture et Vitrerie. Eyrolles, 64-65, I953.

(3) Gilles (G.) et Potapov (B.). - Peinture et Vernissage du bois. Cah. Cent. Techn. Bois, 29, 5I-55, I958. 